\title{
Very low-calorie ketogenic diet (VLCKD): indicazioni ed efficacia nel trattamento dell'obesità
}

\author{
Walter Currenti ${ }^{1} \cdot$ Fabio Galvano $^{1}$
}

Accettato: 3 luglio 2020 / Pubblicato online: 24 novembre 2020

(c) The Author(s) 2020

Sommario La very low-calorie ketogenic diet (VLCKD) è un protocollo alimentare fortemente ipocalorico e ipoglucidico che ha il fine di indurre la chetosi. Rispetto a una dieta ipocalorica tradizionale è vantaggiosa sulla fame, sul mantenimento della muscolatura, sull'infiammazione e sul decremento ponderale. Può essere attuata anche mediante l'utilizzo di prodotti sostitutivi in concomitanza a un percorso di educazione alimentare. La VLCKD rappresenta una valida opzione terapeutica nel trattamento dell'obesità.

Parole chiave Obesità · Chetosi · VLCKD · Pasti sostitutivi · Dieta chetogenica

\section{Introduzione}

Sovrappeso e obesità rappresentano oggi il principale problema di salute pubblica mondiale poiché risultano essere associati a numerose patologie come il diabete mellito di tipo 2, l'ipertensione, la dislipidemia, le malattie cerebrocardiovascolari e oncologiche con conseguente riduzione sia della qualità che dell' aspettativa di vita [1]. Il peso globale dell'obesità è aumentato nel corso del secolo scorso, registrando quasi 2 miliardi di persone in sovrappeso, di cui oltre 600 milioni obese; numeri ancor più allarmanti se si considera il trend in perenne ascesa e il fatto che solo nel 2010

Proposto da Aldo E. Calogero.

Materiale elettronico supplementare La versione elettronica di questo articolo (https://doi.org/10.1007/s40619-020-00796-y) contiene materiale supplementare, disponibile per gli utenti autorizzati.

$凶$ F. Galvano

fgalvano@unict.it

1 Dipartimento di Scienze Biomediche e Biotecnologiche, Università di Catania, Catania, Italia l'eccesso ponderale ha causato 3,4 milioni di morti nel mondo. Tuttavia, si stima che un semplice decremento ponderale pari al 5-10\% del peso corporeo, sia già associato a un significativo beneficio clinico su gran parte delle complicanze correlate all'obesità [2].

Sebbene diversi studi clinici abbiano dimostrato come i cambiamenti dello stile di vita, l'intervento farmacologico e la chirurgia bariatrica favoriscano il calo ponderale, la maggior parte dei pazienti non è in grado di raggiungere il decremento sperato né tantomeno riuscire a mantenerlo nel lungo termine [3]. Nello specifico, molti farmaci attualmente in uso nella pratica clinica risultano essere costosi e non privi di effetti collaterali [4], mentre la chirurgia bariatrica, per quanto estremamente efficace in termini di calo ponderale e di remissione della sindrome metabolica, non è esente da rischi, implica costi elevati ed è indicata solo per una minoranza di pazienti obesi [5].

Per quanto riguarda l'approccio dieto-terapeutico d'elezione per il trattamento dell'obesità, in letteratura l'argomento risulta essere piuttosto controverso; infatti, ad oggi non vi sono dati definitivi su quale sia il protocollo dietetico più efficace [6]. La strategia più comunemente accreditata si basa sulla prescrizione di un regime alimentare globalmente ipocalorico caratterizzato da relativamente alti quantitativi di carboidrati complessi e ridotti quantitativi di grassi totali. Secondo alcuni studi, queste diete a basso tenore di lipidi producono solo modeste perdite di peso e nel lungo termine mostrano elevati tassi di drop-out [7]. In effetti, l'adesione di individui obesi a un'alimentazione ricca di carboidrati e a basso contenuto di grassi è spesso un problema perché la maggior parte ha dimostrato di avere preferenze dietetiche per alimenti con un ricco contenuto di grassi [8]. Un altro problema è che, in generale, gli obesi preferiscono cibi altamente processati contenenti zuccheri semplici piuttosto che carboidrati complessi e fibrosi; quindi, una dieta che pone 
un forte veto per gli alimenti lipidici potrebbe incoraggiare il consumo di zuccheri e carboidrati raffinati che possono peggiorare l'eccesso ponderale oltre che la dislipidemia, specialmente negli individui insulino-resistenti [9]. Infine, è interessante notare come le ultime evidenze scientifiche abbiano trovato un' associazione positiva tra introito di carboidrati e rischio di mortalità totale mentre, all'opposto, un maggiore apporto di grassi totali e, in particolar modo, polinsaturi risultava essere associato a una minore mortalità [10], mettendo in discussione il dogmatico modello calorico dell'obesità, affiancando ad esso quello che la letteratura scientifica definisce "the carbohydrate-insulin model of obesity" [11].

Queste ultime evidenze scientifiche hanno fornito terreno fertile per la riscoperta della dieta chetogenica, un regime alimentare la cui origine risale alla notte dei tempi. Infatti, l'assunzione che uno stato di chetosi potesse influenzare l'organismo e addirittura essere terapeutico per alcuni stati patologici deriva dalle iniziali rilevazioni risalenti a millenni di anni fa sugli effetti del digiuno, condizione chetogenica per eccellenza. Fu però a partire dal 1920 che si cominciò a parlare di vera e propria dieta chetogenica, quando si scoprì che eliminando $i$ carboidrati alimentari si potevano mimare gli effetti metabolici del digiuno e che tale approccio alimentare potesse essere utilizzato in ambito pediatrico per il trattamento delle epilessie farmaco-resistenti. Fu poi lo studioso George Blackburn a introdurre il concetto di Protein-Sparing Modified Fast (PSMF), un regime dietetico fortemente ipocalorico principalmente basato su un sufficiente apporto di proteine e di acidi grassi essenziali tale da permettere un rapido decremento ponderale preservando al contempo la massa muscolare. Ad oggi, la very low-calorie ketogenic diet (VLCKD) rappresenta un'opzione terapeutica valida in numerosi ambiti clinici: obesità in presenza di comorbidità, obesità grave, steatosi epatica, sindrome dell'ovaio policistico, epilessia farmaco-resistente, emicrania e nella gestione preoperatoria in chirurgia bariatrica [12].

La VLCKD simula gli effetti del digiuno attraverso una drastica restrizione dell'apporto giornaliero di carboidrati $(<50 \mathrm{~g} / \mathrm{die})$, con un relativo incremento proporzionale di grassi e proteine e un apporto calorico totale di circa 800 $\mathrm{kcal} /$ die [13]. Bisogna inoltre evidenziare che la VLCKD non è una dieta iperproteica poiché l'apporto giornaliero di proteine raccomandato ammonta a circa $1,2-1,5 \mathrm{~g} / \mathrm{kg}$ di peso corporeo ideale [14].

\section{La chetosi fisiologica}

Nel contesto di una dieta composta da tutti i macronutrienti, l'ossidazione mitocondriale dell' acetil-CoA dipende dai livelli di ossalacetato proveniente soprattutto dalla carbossilazione dell'acido piruvico. Acetil-CoA e ossalacetato si condensano formando citrato che viene consumato nei mitocondri mediante la via ossidativa del ciclo di Krebs. Durante un breve periodo di digiuno (1-3 giorni) o di riduzione dei carboidrati, il glucosio plasmatico è mantenuto dai meccanismi di glicogenolisi e di gluconeogenesi indotte dal glucagone prevalentemente a carico degli amminoacidi. Se questa condizione nutrizionale dovesse protrarsi, glucagone, epinefrina e cortisolo stimolano in maniera massiccia la mobilizzazione degli acidi grassi a partire dai trigliceridi del tessuto adiposo mediante attivazione della lipoproteinlipasi. Gli acidi grassi rilasciati subiscono b-ossidazione nel fegato per formare Acetil-CoA, determinando uno sbilanciamento tra produzione di piruvato, ossalacetato e quella di Acetil CoA. Nelle cellule epatiche l'eccesso di Acetil-CoA viene trasformato nei tre corpi chetonici acetoacetato, $3(\beta)$ idrossibutirato (BHB) e acetone, utilizzati dai tessuti o eliminati successivamente con le urine e, in parte, con il respiro. I corpi chetonici, a differenza degli acidi grassi, riescono a oltrepassare la barriera ematoencefalica divenendo un combustibile alternativo al glucosio per il sistema nervoso centrale.

Già il premio Nobel per la medicina Hans Krebs definì "fisiologica" la chetosi nutrizionale, poiché si tratta di un meccanismo biochimico insito nel metabolismo dell'uomo, affinatosi nel corso dell'evoluzione per contrastare la carenza di cibo e ben diverso dalla condizione di chetoacidosi. Infatti, durante la chetosi nutrizionale la chetonemia si stabilizza intorno a concentrazioni comprese nel range 2-4 $\mathrm{mM} / \mathrm{L}$ non superando mai concentrazioni pari a $7-8 \mathrm{mM} / \mathrm{L}$ e il $\mathrm{pH}$ rimane stabilmente entro livelli assolutamente fisiologici. Invece, nei pazienti diabetici la mancanza assoluta di insulina (tipica del DMT1) o la sua inefficacia (DMT2 scompensato) determina l'impossibilità dei tessuti extraepatici di utilizzare il glucosio e la contemporanea gluconeogenesi epatica, proteolisi e lipolisi. L'iperglicemia induce glicosuria e, dunque, diuresi osmotica; la forte diminuzione della riserva alcalina, unita all'ipovolemia e alla sofferenza renale, porta ad acidosi. Durante la chetoacidosi diabetica i valori di corpi chetonici possono superare i $20 \mathrm{mmol} / \mathrm{L}$.

\section{Meccanismi di azione della VLCKD}

Quando l'organismo viene sottoposto a VLCKD, le concentrazioni ematiche di insulina diminuiscono, mentre il glucagone aumenta per mantenere normali i livelli di glicemia. Si verifica un aumento degli acidi grassi liberi e successivamente, dopo circa 3 giorni, un consistente aumento dei corpi chetonici nel sangue $\geq 4 \mathrm{mmol} / \mathrm{l}$, valore associato a una netta diminuzione della fame. Si pensa che l'effetto anoressizzante di un protocollo chetogenico non derivi solo dall'azione diretta dei corpi chetonici, in particolare dal BHB per sé, ma anche dall'effetto saziante degli alimenti proteici, oltre 
che dall'azione su alcuni ormoni implicati nella regolazione dell'appetito come il neuropeptide Y, la colecistochinina e la ghrelin [15]. L'inappetenza indotta dai corpi chetonici viene spesso accompagnata da un'azione euforizzante e stabilizzante l'umore, sostenuta da un aumento delle catecolammine, effetti entrambi estremamente utili per aumentare la compliance dietetica dei pazienti. L'effetto anoressizzante non sembrerebbe essere l'unico motivo dello spiccato effetto di una VLCKD sul calo ponderale. Infatti, nelle fasi iniziali di una dieta molto povera in carboidrati, il corpo ricava glucosio mediante gluconeogenesi a discapito prevalentemente delle proteine alimentari e tissutali e, in minor parte, dal glicerolo risultante dalla completa idrolisi dei trigliceridi adipocitari.

Molti autori ipotizzano che l'utilizzo a scopo energetico delle proteine nella VLCKD sia un processo dispendioso per l'organismo e che, quindi, possa indurre a un aumento del dispendio calorico. Al costo energetico della gluconeogenesi bisogna aggiungere l'azione dinamico-specifica degli alimenti. Tale parametro calcola la spesa energetica che l'organismo deve sostenere per assorbire e metabolizzare i nutrienti, che risulta essere in generale superiore per gli alimenti proteici [16].

L'assenza di carboidrati, determinando elevati livelli di glucagone e bassi livelli di insulina, induce l'organismo a ridurre i meccanismi di lipogenesi favorendo quelli lipolitici, con conseguente massivo utilizzo dei trigliceridi adipocitari a fini energetici e, nel medio-lungo termine, effetto risparmiatore sulla massa muscolare [17]. A prova di ciò vi è la drastica diminuzione del quoziente respiratorio a riposo.

I ridotti apporti di carboidrati e, conseguentemente, i bassi livelli di insulina secreta, risultano essere determinanti sulla riduzione dei trigliceridi ematici, sull'abbassamento del colesterolo totale e sull' aumento del colesterolo HDL [18]. L'insulina, infatti, ha anche un'azione stimolante sulla HMGCoA reduttasi che è deputata alla produzione del colesterolo. La minore insulinemia indotta da un protocollo VLCKD potrebbe essere di estremo beneficio per la condizione di insulino-resistenza comunemente presente nei pazienti obesi. Una VLCKD, infatti, può migliorare i parametri dell'omeostasi glicemica e l'utilizzo di farmaci ipoglicemizzanti in maniera indipendente dal calo ponderale [19].

\section{Indicazioni e controindicazioni cliniche della dieta chetogenica}

Per gli effetti precedentemente analizzati, la VLCKD si erge a buon titolo come una strategia valida e alternativa per indurre un calo ponderale nel paziente obeso [20]. Sono ormai numerosi gli studi scientifici che hanno mostrato come una VLCKD riesca a indurre una riduzione del grasso viscerale e un complessivo miglioramento dei parametri metabolici e dei marker infiammatori sistemici [19]. Queste proprietà rendono la VLCKD particolarmente indicata per l'obesità severa e per l'obesità con comorbidità. La drastica riduzione dei carboidrati alimentari induce immediati vantaggi sul controllo glicemico, sulla resistenza insulinica e sul funzionamento delle $\beta$-cellule pancreatiche, al punto che sono stati rilevati casi di completa remissione dal diabete mellito di tipo 2, particolarmente in pazienti neo-diagnosticati sottoposti a VLCKD.

La carenza di carboidrati consente, inoltre, una deplezione del contenuto intraepatico di trigliceridi, l'inibizione della gluconeogenesi e un incremento della chetogenesi epatica; tutti effetti benefici nei confronti di un'eventuale steatosi epatica. Grazie all'efficacia di una VLCKD nell'indurre rapidamente una riduzione del volume epatico, a partire dal 2016 la VLCKD è entrata a far parte del protocollo per il calo ponderale preoperatorio nel paziente candidato all'intervento di chirurgia bariatrica. È stato dimostrato che una VLCKD preoperatoria può determinare riduzione dei tempi di degenza, migliore stabilizzazione dei livelli di emoglobina postoperatori e minor tempo di cicatrizzazione della ferita chirurgica [21].

La VLCKD consente, inoltre, di preservare la massa muscolare riducendo il rischio di sarcopenia e della conseguente riduzione del metabolismo basale durante il protocollo di restrizione calorica.

Altre indicazioni e le controindicazioni assoluta sono raffigurate rispettivamente nella Tabella 1 e nella Tabella 2.

\section{Elaborazione di un protocollo}

Secondo le linee guida redatte dal Panel on Nutrition, Novel Foods and Food Allergens della European Food Safety Authority, la VLCKD è considerata una terapia dietetica suddivisa in fasi e caratterizzata da un basso apporto sia carboidrati che di calorie ( $<800 \mathrm{kcal} / \mathrm{die})$. Nello specifico, un piano VLCKD prevede in media un apporto glucidico tra i 20 e i $60 \mathrm{~g} /$ die ottenuto da alimenti vegetali, un apporto proteico tra $1,2-1,5 \mathrm{~g} / \mathrm{kg}$ di peso corporeo ideale da fonti alimentari ad alto valore biologico per preservare la massa muscolare e, infine, tra i 15 e i $30 \mathrm{~g}$ di apporto lipidico ottenuto prevalentemente da olio extravergine di oliva e da acidi grassi polinsaturi della serie omega 3. Al fine di poter soddisfare queste stringenti prescrizioni alimentari, specie nella fase iniziale di un piano VLCKD, risulta utile l'utilizzo di pasti sostitutivi. Sono di comune utilizzo i preparati a base di proteine ad alto valore biologico, provenienti prevalentemente dal siero del latte, dalle uova o, per pazienti vegani, dai legumi. Queste formulazioni contengono in genere 100 $150 \mathrm{kcal}$ di cui $18 \mathrm{~g}$ proteine, $4 \mathrm{~g}$ carboidrati e $3 \mathrm{~g}$ di grassi [14]. 
Tabella 1 Indicazioni alla VLCKD (da [14])

Indicazioni forti

- Obesità severa

- Pre-chirurgia bariatrica

- Obesità sarcopenica

- Obesità associata a comorbidità (ipertensione arteriosa, diabete mellito di II tipo, dislipidemia, sindrome delle apnee notturne di tipo ostruttivo, osteopatie)

- Obesità pediatrica associata a epilessia e $\backslash$ o elevata insulino resistenza o comorbidità, non responsivo a dieta tradizionale

Indicazioni deboli

- Obesità associata a: disbiosi, elevati livelli LDL e $\backslash$ o bassi HDL, aterosclerosi, insufficienza cardiaca, NAFLD, PCOS

- Obesità secondaria a ipogonadismo

- Malattie neurodegenerative associate a obesità sarcopenica

Tabella 2 Controindicazioni assolute alla VLCKD (da [14])

- Gravidanza e allattamento

- Diabete di tipo 1, LADA, diabete tipo 2 con severa disfunzione beta-cellulare, uso concomitante di SGLT2 inibitori

- Angina instabile, recente ictus o infarto del miocardio ( $<12$ mesi), aritmia cardiaca; Insufficienza renale moderata-severa, insufficienza epatica, insufficienza cardiaca (NYHA III-IV), insufficienza respiratoria

- Disturbi del comportamento alimentare, disturbi psichici, abuso di alcol o altre sostanze

- Malattie rare: porfiria, deficit di carnitina, deficit di palmitoiltransferasi, deficit di carnitina-acetilcarnitina translocasi, disordini della beta-ossidazione mitocondriale degli acidi grassi, deficit di piruvato carbossilasi

Sebbene i preparati da disciogliere in acqua siano i più utilizzati per la loro praticità d'uso, ad oggi il mercato propone numerose tipologie di pasti sostitutivi dalle caratteristiche organolettiche sempre più invitanti tra cui prodotti da forno, barrette e zuppe. Una volta stabiliti i macronutrienti da somministrare al paziente, il protocollo VLCKD si suddivide principalmente in tre macro-fasi; fase della chetosi, fase di reintroduzione alimentare e fase di mantenimento (Fig. 1). Nella prima fase l'obiettivo è quello di indurre la chetosi nel paziente. Affinché questo avvenga e vi sia già un evidente decremento ponderale, l'alimentazione del paziente potrà essere esclusivamente basata su 4-6 pasti sostitutivi giornalieri accompagnati da verdure. Nelle settimane successive si va a introdurre un alimento proteico convenzionale (carne/pesce/uova) prima a un solo pasto principale e poi a entrambi i pasti principali, in modo da ridurre l'utilizzo dei prodotti sostitutivi e avviare il paziente verso una dieta più varia. Questa prima fase, la cui durata si stima essere in genere tra le 8-12 settimane, rappresenta il momento più rilevante del percorso dietetico e dovrebbe indurre una perdita di peso pari all' $80-85 \%$ del calo ponderale stabilito. Durante tutta la fase di chetosi, la dieta deve essere adeguatamente supplementata con integratori a base di potassio, calcio, magnesio, sodio, vitamine e omega-3 per sopperire alla mancanza di numerosi micronutrienti e all'incremento della diuresi indotto dalla VLCKD. Per monitorare l'efficacia e l'aderenza alla dieta è consigliabile rilevare i corpi chetonici a livello urinario o capillare.
Dopo la prima fase strettamente chetogenica a bassissimo contenuto calorico, vengono leggermente alzate le calorie (800-1500 kcal \die) e reintrodotti gradualmente gli alimenti glucidici partendo da quelli a indice glicemico più basso (frutta, latticini), proseguendo poi con i legumi e, infine, aggiungendo pane, pasta e cereali di vario genere. Questa fase deve essere adeguatamente affiancata da un percorso di educazione alimentare che permetta al paziente di reintrodurre gli alimenti in maniera graduale e consapevole dei principi nutrizionali in essi contenuti. Il paziente deve essere, inoltre, introdotto a un progressivo incremento dell'attività fisica. Questa seconda fase si conclude quando il $100 \%$ del calo ponderale stabilito è stato ottenuto e tutti gli alimenti sono stati inseriti nella dieta. L'obiettivo finale, pertanto, è quello di condurre il paziente verso una dieta equilibrata sullo stile mediterraneo con un apporto calorico tra le 1500-2000 kcal che gli permetta di mantenere i risultati nel lungo termine (fase di mantenimento).

\section{Effetti indesiderati VLCKD}

La VLCKD deve essere considerata una vera e propria terapia e, come tale, può essere considerata sicura solo sotto un'attenta sorveglianza medica. Al fine di intraprendere un percorso alimentare di tale genere è necessario effettuare esami ematici e strumentali con periodicità. L'analisi della composizione corporea mediante bioimpedenziometria (BIA) potrebbe rivelarsi un'analisi interessante alla quale 
Fig. 1 Le fasi di un protocollo VLCKD (da [13])

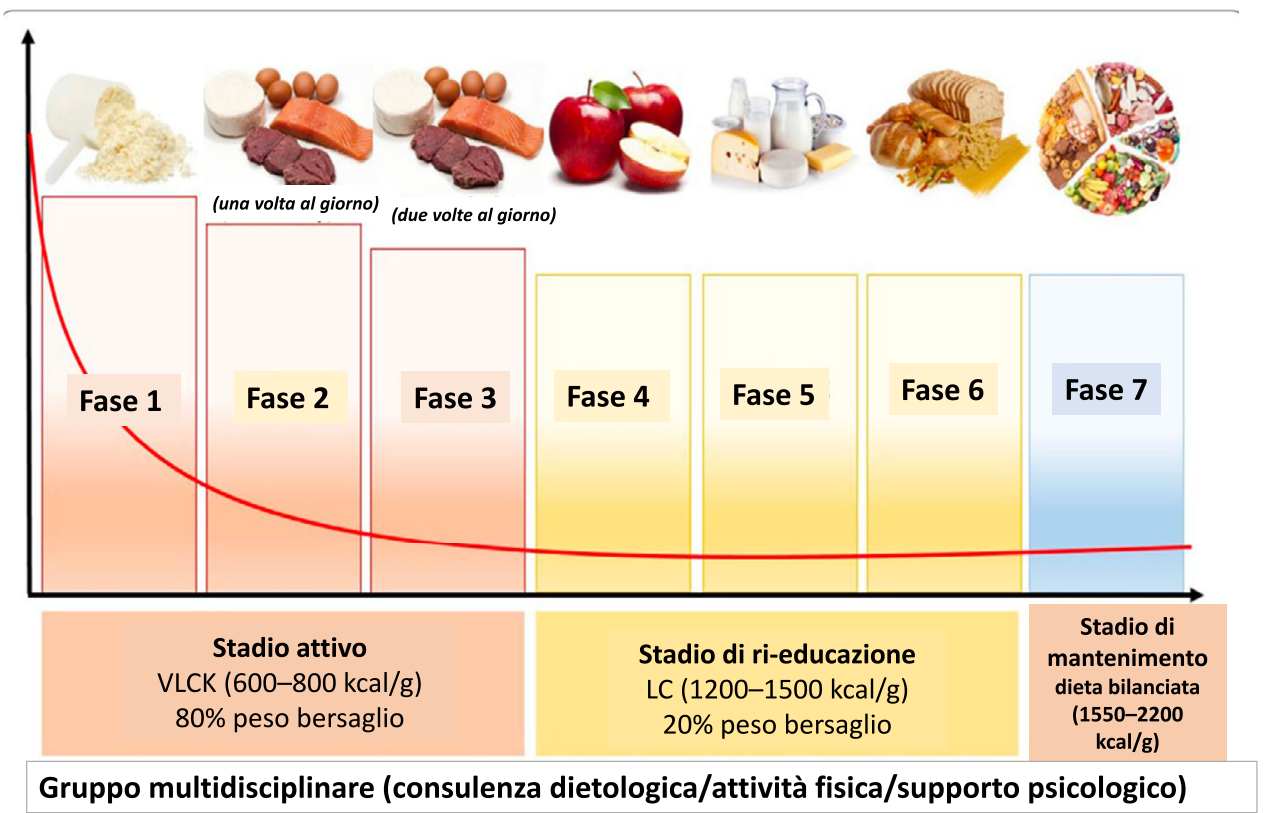

sottoporre con periodicità il paziente al fine di rilevare un eventuale eccessivo cambiamento dell' acqua corporea totale e valutare l'effetto anti-catabolico della chetosi sulla massa muscolare dell'individuo.

Gli effetti indesiderati più comuni durante una VLCKD ben monitorata risultano essere blandi e facilmente reversibili. Le iniziali manifestazioni cliniche sono la cefalea e la debolezza riportata da circa un terzo dei pazienti che, però, tende a scomparire entro le 72 ore con l'innesco dello stato di chetosi. L'alitosi invece è una manifestazione attribuibile all'acetone che viene eliminato per via respiratoria. Per quanto riguarda l'alvo vi è grande variabilità e di frequente si manifesta l'alternanza di stipsi a diarrea. La disidratazione, invece, può essere ingente, specie nelle fasi iniziali del protocollo, pertanto il paziente deve essere consapevole dell'importanza che ricopre un corretto apporto idrico $(>2$ $\mathrm{L} \backslash$ die) e di un'integrazione salina.

\section{Conclusioni}

La VLCKD è un protocollo alimentare fortemente ipocalorico, caratterizzato da un ridotto apporto glucidico $(<50 \mathrm{~g}) \mathrm{e}$ un adeguato apporto di proteine ad alto valore biologico tale da preservare la massa muscolare nonostante il repentino calo ponderale. L'induzione dello stato di chetosi permette di ottenere un maggiore effetto saziante nonostante l'apporto calorico estremamente basso ( $<800 \mathrm{kcal})$ oltre che usufruire dei benefici indotti dall'utilizzo dei corpi chetonici a fini energetici, specie sull'infiammazione e sul grasso viscerale. Per tali motivi, la VLCKD rappresenta una valida opzione terapeutica nel trattamento dell'obesità.
Funding Note Open access funding provided by Università degli Studi di Catania within the CRUI-CARE Agreement.

Conflitto di interesse Gli autori Walter Currenti e Fabio Galvano dichiarano di non avere conflitti di interesse.

Consenso informato Lo studio presentato in questo articolo non ha richiesto sperimentazione umana.

Studi sugli animali Gli autori di questo articolo non hanno eseguito studi sugli animali.

Nota della casa editrice Springer Nature rimane neutrale in riguardo alle rivendicazioni giurisdizionali nelle mappe pubblicate e nelle affiliazioni istituzionali.

Open Access This article is licensed under a Creative Commons Attribution 4.0 International License, which permits use, sharing, adaptation, distribution and reproduction in any medium or format, as long as you give appropriate credit to the original author(s) and the source, provide a link to the Creative Commons licence, and indicate if changes were made. The images or other third party material in this article are included in the article's Creative Commons licence, unless indicated otherwise in a credit line to the material. If material is not included in the article's Creative Commons licence and your intended use is not permitted by statutory regulation or exceeds the permitted use, you will need to obtain permission directly from the copyright holder. To view a copy of this licence, visit http://creativecommons.org/licenses/by/4.0/.

\section{Bibliografia}

1. Di Angelantonio E et al (Global BMI Mortality Collaboration) (2016) Body-mass index and all-cause mortality: individualparticipant-data meta-analysis of 239 prospective studies in four continents. Lancet 388(10046):776-786

2. Ma C, Avenell A, Bolland M et al (2017) Effects of weight loss interventions for adults who are obese on mortality, cardiovascular disease, and cancer: systematic review and meta-analysis. BMJ 359:j4849 
3. Siraj ES, Williams KJ (2015) Another agent for obesity-will this time be different? N Engl J Med 373(1):82-83

4. Patel DK, Stanford FC (2018) Safety and tolerability of newgeneration anti-obesity medications: a narrative review. Postgrad Med 130(2):173-182

5. Pories WJ (2008) Bariatric surgery: risks and rewards. J Clin Endocrinol Metab 93(11 Suppl 1):S89-S89

6. Nordmann AJ, Nordmann A, Briel M et al (2006) Effects of lowcarbohydrate vs. low-fat diets on weight loss and cardiovascular risk factors: a meta-analysis of randomized controlled trials. Arch Intern Med 166:285-290

7. Brehm BJ, Seeley RJ, Daniels SR et al (2003) Randomized trial comparing a very low carbohydrate diet and a calorie-restricted low fat diet on body weight and cardiovascular risk factors in healthy women. J Clin Endocrinol Metab 88:1617-1623

8. Drewnowski A, Krahn DD, Demitrack MA et al (1992) Taste responses and preferences for sweet high-fat foods: evidence for opioid involvement. Physiol Behav 51:371-379

9. Paoli A, Rubini A, Volek JS et al (2013) Beyond weight loss: a review of the therapeutic uses of very-low-carbohydrate (ketogenic) diets. Eur J Clin Nutr 67:789-796

10. Dehghan M, Mente A, Zhang X et al (2017) Associations of fats and carbohydrate intake with cardiovascular disease and mortality in 18 countries from five continents (PURE): a prospective cohort study. Lancet 390(10107):2050-2062

11. Ludwig DS, Ebbeling CB (2018) The carbohydrate-insulin model of obesity: beyond "calories in, calories out". JAMA Intern Med 178(8):1098-1103

12. Pezzana AM, Fatati G, Caregaro Negrin L et al (2014) La dieta chetogenica-Fondazione ADI: position paper. ADI 6:38-43
13. Muscogiuri G, Barrea L, Laudisio D et al (2019) The management of very low-calorie ketogenic diet in obesity outpatient clinic: a practical guide. J Transl Med 17:356

14. Caprio M, Infante M, Moriconi E et al (2019) Very-low-calorie ketogenic diet (VLCKD) in the management of metabolic diseases: systematic review and consensus statement from the Italian Society of Endocrinology (SIE). J Endocrinol Invest 42(11):1365-1386

15. Sumithran P, Prendergast LA, Delbridge E et al (2013) Ketosis and appetite-mediating nutrients and hormones after weight loss. Eur J Clin Nutr 67:759-764

16. Manninen AH (2006) Metabolic advantage of low-carbohydrate diets: a calorie is still not a calorie. Am J Clin Nutr 83:1442-1443

17. Tinsley GM, Willoughby DS (2016) Fat-free mass changes during ketogenic diets and the potential role of resistance training. Int $\mathbf{J}$ Sport Nutr Exerc Metab 26(1):78-92

18. Sharman MJ, Kraemer WJ, Love DM et al (2002) A ketogenic diet favorably affects serum biomarkers for cardiovascular disease in normal-weight men. J Nutr 132:1879-1885

19. Paoli A (2014) Ketogenic diet for obesity: friend or foe? Int $\mathbf{J}$ Environ Res Public Health 11(2):2092-2107

20. Castellana M, Conte E, Cignarelli A et al (2020) Efficacy and safety of very low calorie ketogenic diet (VLCKD) in patients with overweight and obesity: a systematic review and meta-analysis. Rev Endocr Metab Disord 21(1):5-16

21. Albanese A, Prevedello L, Markovich M et al (2019) Pre-operative very low calorie ketogenic diet (VLCKD) vs. very low calorie diet (VLCD): surgical impact. Obes Surg 29(1):292-296 